\title{
Drug-induced gingival hyperplasia: a retrospective study using spontaneous reporting system databases
}

Haruna Hatahira', Junko Abe ${ }^{1,2}$, Yuuki Hane ${ }^{1}$, Toshinobu Matsui ${ }^{1}$, Sayaka Sasaoka', Yumi Motooka', Shiori Hasegawa', Akiho Fukuda', Misa Naganuma', Tomofumi Ohmori ${ }^{1,3}$, Yasutomi Kinosada ${ }^{4}$ and Mitsuhiro Nakamura ${ }^{1 *}$ (D)

\begin{abstract}
Background: Drug-induced gingival hyperplasia (DIGH) causes problems with chewing, aesthetics, and pronunciation, and leads to the deterioration of the patient's quality of life (QOL). Thus, the aim of this study was to evaluate the incidence of DIGH using spontaneous reporting system (SRS) databases.

Methods: We analyzed reports of DIGH from SRS databases and calculated the reporting odds ratios (RORs) of suspected drugs (immunosuppressants, calcium channel blockers, and anticonvulsants). The SRS databases used were the US Food and Drug Administration (FDA) Adverse Event Reporting System (FAERS) and the Japanese Adverse Drug Event Report (JADER) database. With the data, we evaluated the time-to-onset profile and the hazard type using the Weibull shape parameter (WSP). Furthermore, we used the association rule mining technique to discover undetected relationships such as possible risk factors.

Results: The FAERS contained 5,821,716 reports. The RORs (95\% confidence interval: Cl) for cyclosporine, everolimus, sirolimus, mycophenolate mofetil, amlodipine, nifedipine, carbamazepine, clobazam, levetiracetam, phenobarbital, phenytoin, primidone, topiramate, and valproic acid, were 39.4 (95\% Cl: 30.3-51.2), 4.2 (1.7-10.0), 6.6 (2.5-17.7), 13.1 (7.2-23.2), 94.8 (80.0-112.9), 57.9 (35.7-94.0), 15.1 (10.3-22.3), 65.4 (33.8-126.7), 6.5 (3.6-11.8), 19.7 (8.8-44.0), 65.4 (52.4-82.9), 56.5 (21.1-151.7), 2.9 (1.1-7.7), and 17.5 (12.6-24.4), respectively. The JADER database contained 430,587 reports. The median time-to-onset of gingival hyperplasia values for immunosuppressants, calcium channel blockers, and anticonvulsants use were 71, 262, and 37 days, respectively. Furthermore, the 95\% Cl of the WSP $\beta$ for anticonvulsants was over and excluded 1, which meant that they were wear-out failure type.
\end{abstract}

Conclusions: Our results suggest that DIGH monitoring of patients administered immunosuppressants, calcium channel blockers, or anticonvulsants is important. We demonstrated the potential risk of DIGH following the long-term use of calcium channel blocker over approximately 260 days. Based on the results of the association rule mining approach, patients with intellectual disability who are administered phenytoin should be monitored carefully. We recommend that patients who experience symptoms related to DIGH should be closely monitored.

Keywords: Drug-induced gingival hyperplasia (DIGH), JADER, FAERS, Time-to-onset analysis, Association rule mining technique, Spontaneous reporting system

\footnotetext{
* Correspondence: mnakamura@gifu-pu.ac.jp

'Laboratory of Drug Informatics, Gifu Pharmaceutical University, 1-25-4

Daigaku-nishi, Gifu 501-1196, Japan

Full list of author information is available at the end of the article
} 


\section{Background}

Drug-induced gingival hyperplasia (DIGH) is a periodontal side effects of certain drugs, causing swelling, bleeding, and problems with chewing, aesthetics, and pronunciation. In more severe cases, it can cause high mobility and detachment of the teeth due to alveolar bone absorption. All of these effects lead to the deterioration of the patient's quality of life (QOL). More than 20 drugs are associated with DIGH [1], principal among them are immunosuppressants, calcium channel blockers, and anticonvulsants [1]. Dongali-Bagtzoglow [1] reported that $>70,6-15$, and $50 \%$ of DIGH incidences were observed with cyclosporine (CsA; an immunosuppressant), nifedipine (a calcium channel blocker), and phenytoin (an anticonvulsant), respectively.

Since DIGH is a rare adverse event, epidemiologic research is difficult to perform. Spontaneous reporting systems (SRSs) are useful for the detection of rare adverse events and have been recognized as primary tools for pharmacovigilance that reflect the realities of clinical practice. The main aim of regulatory authorities is to collect and store safety reports for monitoring community health. Several pharmacovigilance indexes including the reporting odds ratio (ROR), were developed to evaluate drug-associated adverse events determined though SRS data. The concept of disproportionate analysis of ROR is common in the conventional analysis of SRSs, which attempts to quantify the degree of "unexpectedness" of a drug to adverse event association.

Recently, analysis of time-to-onset data has been proposed as a new method to detect signals for adverse events in SRS. To the best of our knowledge, analyses of the time-to-onset for DIGH using the Japanese Adverse Drug Event Report (JADER) database are rare. Association rule mining has been proposed as an analytical approach in order to study rare adverse drug events, and is a wellestablished method for discovering undetected relationships such as possible risk factors between variables in huge databases [2-4]. We examined DIGH using both analytical methods, and adjusted for the influence of demography and polypharmacy. This is the first study to evaluate the association between drugs and DIGH using ROR, time-to-onset analysis, and association rule mining. The aims of the study were to obtain new information of risk comparison on drugs or undetected several clinical factor combination, and onset profiles of DIGH for prescription drugs in the real world.

\section{Methods}

\section{Data sources}

The US Food and Drug Administration (FDA) adverse event reporting system (FAERS) is an SRS and the largest and best-known database worldwide. The regulatory authority in Japan, the Pharmaceuticals and Medical
Devices Agency (PMDA), controls the SRS of the JADER database. Adverse events recorded in the FAERS database from January 2004 to June 2014 were downloaded from the FDA website (http://www.fda.gov). Relevant information from the JADER database from April 2004 to November 2016 was downloaded from the PMDA website (http://www.pmda.go.jp). We constructed a database that integrated each FAERS and JADER dataset using the FileMaker Pro 13 (FileMaker Inc.). For duplicate entries, we followed the FDA recommendation (http:// www.fda.gov/Drugs/GuidanceComplianceRegulatoryInformation/Surveillance/AdverseDrugEffects), and adopted the most recent case number to identify duplicate patient reports and excluded them from the analysis.

We analyzed four immunosuppressants (CsA, everolimus, sirolimus, and mycophenolate mofetil), four calcium channel blockers (amlodipine, benidipine, nicardipine and nifedipine), and 11 anticonvulsants (carbamazepine, clobazam, diazepam, gabapentin, levetiracetam, phenobarbital, phenytoin, primidone, topiramate, valproic acid, and zonisamide). For drug definitions, we used both the general and brand names based on the DrugBank 3.0 and 4.0 (Table 1). Drugs in the FAERS were classified into four categories: Primary Suspect drug (PS), Secondary Suspect drug (SS), Concomitant (C), and Interacting (I); according to their anticipated degree of involvement in adverse events. The analysis was restricted to reports where drugs were recorded as PS and SS in the FAERS database. In the "drug information" table of the JADER database, each drug was assigned a code according to its association with adverse drug reactions: "suspected drug," "concomitant drug," or "interacting drug." The analysis was restricted to reports where drugs were recorded as "suspected drugs" in the JADER database.

\section{Definition of DIGH}

The adverse event definitions used in FAERS were those provided by the Medical Dictionary for Regulatory Activities (MedDRA) version 17.1. For the extraction of cases from the FAERS database, we used two preferred terms (PTs), gingival hyperplasia (PT code: 10018283) and gingival hypertrophy (PT code: 10018284). The adverse event definitions used in JADER were those provided by MedDRA version 19.0. In the MedDRA 19.0, the two PTs related to DIGH were combined into "gingival hypertrophy (PT code: 10018284).” Thus, for the extraction of cases from the JADER database, we used the PT gingival hypertrophy (PT code: 10018284).

\section{Data mining}

ROR

For the detection of DIGH, we calculated the ROR as the ratio of the odds of reporting a DIGH adverse event versus all other events for a given drug, compared to the 
Table 1 Brand names of drugs

\begin{tabular}{|c|c|c|}
\hline Generic name ${ }^{a}$ & $\begin{array}{l}\text { Number of brand } \\
\text { name }\end{array}$ & Brand name \\
\hline \multicolumn{3}{|c|}{ Immunosuppressants } \\
\hline Cyclosporine & 6 & Gengraf, Neoral, etc. \\
\hline Everolimus & 1 & Certican \\
\hline Sirolimus & 1 & Rapamune \\
\hline $\begin{array}{l}\text { Mycophenolate } \\
\text { mofetil }\end{array}$ & 2 & Cellcept, Mucoloc \\
\hline \multicolumn{3}{|l|}{$\begin{array}{l}\text { Calcium Channel } \\
\text { Blockers }\end{array}$} \\
\hline Amlodipine & 7 & Amlocard, Amlodis, etc. \\
\hline Benidipine $^{\mathrm{b}}$ & - & - \\
\hline Nicardipine & 3 & Cardene, Cardene IV, etc. \\
\hline Nifedipine & 106 & Adalat, Adalat 10, etc. \\
\hline \multicolumn{3}{|l|}{ Anticonvulsants } \\
\hline Carbamazepine & 29 & $\begin{array}{l}\text { Apo-Carbamazepine, } \\
\text { Atretol, etc. }\end{array}$ \\
\hline Clobazam & 6 & Chlorepin, Clorepin, etc. \\
\hline Diazepam & 116 & Alboral, Aliseum, etc. \\
\hline Gabapentin & 3 & Aclonium, Neurontin, etc. \\
\hline Levetiracetam & 1 & Keppra \\
\hline Phenobarbital & 138 & Adonal, Aephenal, etc. \\
\hline Phenytoin & 130 & Aleviatin, Antisacer, etc. \\
\hline Primidone & 36 & Apo-Primidone, Cyral, etc. \\
\hline Topiramate & 2 & $\begin{array}{l}\text { Topamax, Topamax } \\
\text { Sprinkle, etc. }\end{array}$ \\
\hline Valproic acid & 25 & $\begin{array}{l}\text { Alti-Valproic, Avugane, } \\
\text { etc. }\end{array}$ \\
\hline Zonisamide & 4 & Excegran, Exegram, etc. \\
\hline
\end{tabular}

reporting odds for all other drugs. We detected the signals when the ROR estimated and lower limits of the corresponding $95 \%$ confidence interval $(\mathrm{CI})$ were greater than 1 , and at least 2 cases were required to define the signal $[5,6]$.

\section{Time-to-onset analysis}

Median, quartile, and Weibull shape parameter (WSP) tests were used to evaluate the time-to-onset analysis [7-10]. We analyzed the time the specific adverse event occurred from when the prescription of specific drugs commenced by using the Weibull distribution parameter. We excluded reports that did not have complete adverse event occurrence and prescription start times. The scale parameter $\alpha$ determined the scale of the distribution function while the shape parameter $\beta$ determined the shape of the distribution function. In the analysis of the SRSs, the shape parameter $\beta$ of the Weibull distribution was used to indicate the hazard without reference populations as follows: If the $95 \% \mathrm{CI}$ of $\beta$ included 1 , the hazard was estimated to be constant over time (random failure type). If the lower limit of the $95 \% \mathrm{CI}$ of $\beta$ was greater than 1 , the hazard was considered to increase over time (wear-out failure type). If the upper limit of the $95 \% \mathrm{CI}$ of $\beta$ was less than 1 , the hazard was considered to decrease over time (initial failure type) [11]. The time-to-onset analysis was performed using JMP version 11.0 software (SAS Institute, Cary, NC, USA).

\section{Association rule mining}

The association rule mining approach attempts to evaluate frequent items in databases. Given a set of transactions $\boldsymbol{T}$, an association rule can be expressed as $\mathrm{X} \rightarrow \mathrm{Y}$, where $\mathrm{X}$ and $\mathrm{Y}$ are mutually exclusive sets of items [12-14]. The rule's statistical significance and strength are measured as support and confidence. The support is defined as the percentage of transactions in the data that contain all items in both the antecedent (left hand side) and the consequent (right hand side) of the rule [12-14]. The support indicates how frequently the rule occurs in the transaction and has the following formula:

$$
\text { Support }=\mathrm{P}(\mathrm{X} \cap \mathrm{Y})=\{\mathrm{X} \cap \mathrm{Y}\} /\{\mathrm{D}\}
$$

where $\mathrm{D}$ is total number of transactions in the database. The confidence corresponds to the conditional probability $\mathrm{P}(\mathrm{Y} \mid \mathrm{X})$. It is important for a rule to have a high confidence because it provides an accurate prediction of the association of the items in the rule. The formula for calculating confidence is as follows:

$$
\text { Confidence }=\mathrm{P}(\mathrm{X} \cap \mathrm{Y}) / \mathrm{P}(\mathrm{X})
$$

Lift is the probability of $\mathrm{X}$ and $\mathrm{Y}$ occurring together divided by the multiple of the two individual probabilities for $\mathrm{X}$ and $\mathrm{Y}$; that is,

$$
\text { Lift }=\mathrm{P}(\mathrm{X} \cap \mathrm{Y}) / \mathrm{P}(\mathrm{X}) \mathrm{P}(\mathrm{Y})
$$

Since P (Y) appears in the denominator of the lift equation, the lift can be considered to be the confidence divided by $\mathrm{P}(\mathrm{Y})$. The lift can be evaluated as follows: lift $=1,>1$, and $<1$ if $\mathrm{X}$ and $\mathrm{Y}$ are independent, positively correlated, and negatively correlated, respectively. We performed these analyses using the apriori function of the arules library in the arules package $\mathrm{R}$ version 3.3.2 software [15].

\section{Results}

The FAERS database contained 5,821,716 reports that were submitted between January 2004 and June 2014 . After deleting the duplicate reports, 4,551,642 reports were analyzed. The number of case reports and the RORs are summarized in Table 2. The RORs for cases 
Table 2 Number of reports and the reporting odds ratio for gingival hyperplasia by drugs

\begin{tabular}{|c|c|c|c|c|c|c|c|c|}
\hline \multirow[t]{2}{*}{ Drug } & \multicolumn{4}{|l|}{ Total } & \multicolumn{4}{|c|}{$\underline{\text { Suspected Drug }^{\mathrm{a}}, \mathrm{b}}$} \\
\hline & Total & Case & $\mathrm{ROR}$ & $(95 \% \mathrm{Cl})$ & Total & Case & $\mathrm{ROR}$ & $(95 \% \mathrm{Cl})$ \\
\hline FAERS & $4,551,642$ & 628 & & & & & & \\
\hline \multicolumn{9}{|l|}{ Immunosuppressants } \\
\hline Cyclosporine & 20,578 & 66 & 25.9 & $(20.1-33.5)$ & 12,693 & 62 & 39.4 & $(30.3-51.2)$ \\
\hline Everolimus & 9292 & 6 & 4.7 & $(2.1-10.5)$ & 8756 & 5 & 4.2 & $(1.7-10.0)$ \\
\hline Sirolimus & 5607 & 5 & 6.5 & $(2.7-15.7)$ & 4415 & 4 & 6.6 & $(2.5-17.7)$ \\
\hline Mycophenolate mofetil & 20,212 & 30 & 11.3 & $(7.8-16.3)$ & 6213 & 11 & 13.1 & $(7.2-23.2)$ \\
\hline \multicolumn{9}{|l|}{ Calcium Channel Blockers } \\
\hline Amlodipine & 96,153 & 195 & 20.9 & $(17.7-24.8)$ & 18,509 & 174 & 94.8 & $(80.0-112.9)$ \\
\hline Benidipine & 423 & 0 & - & & 29 & 0 & - & \\
\hline Nicardipine & 1838 & 1 & $-^{c}$ & & 674 & 1 & $-^{c}$ & \\
\hline Nifedipine & 18,542 & 23 & 9.3 & $(6.1-14.1)$ & 2202 & 17 & 57.9 & $(35.7-94.0)$ \\
\hline \multicolumn{9}{|l|}{ Anticonvulsants } \\
\hline Carbamazepine & 24,644 & 31 & 9.6 & $(6.7-13.7)$ & 13,494 & 27 & 15.1 & $(10.3-22.3)$ \\
\hline Clobazam & 3155 & 11 & 25.8 & $(14.2-46.9)$ & 1020 & 9 & 65.4 & $(33.8-126.7)$ \\
\hline Diazepam & 36,751 & 9 & 1.8 & $(0.9-3.5)$ & 11,581 & 3 & 1.9 & $(0.6-5.9)$ \\
\hline Gabapentin & 71,069 & 11 & 1.1 & $(0.6-2.0)$ & 17,372 & 2 & 0.8 & $(0.2-3.3)$ \\
\hline Levetiracetam & 2630 & 14 & 39.6 & $(23.3-67.4)$ & 12,437 & 11 & 6.5 & $(3.6-11.8)$ \\
\hline Phenobarbital & 7717 & 18 & 17.4 & $(10.9-27.8)$ & 2235 & 6 & 19.7 & $(8.8-44.0)$ \\
\hline Phenytoin & 20,522 & 95 & 39.5 & $(31.8-49.2)$ & 10,865 & 85 & 65.4 & $(52.4-82.9)$ \\
\hline Primidone & 2644 & 10 & 27.9 & $(14.9-52.3)$ & 520 & 4 & 56.5 & $(21.1-151.7)$ \\
\hline Topiramate & 23,635 & 7 & 2.2 & $(1.0-4.6)$ & 10,066 & 4 & 2.9 & $(1.1-7.7)$ \\
\hline Valproic acid & 34,487 & 43 & 9.6 & $(7.1-13.1)$ & 16,258 & 37 & 17.5 & $(12.6-24.4)$ \\
\hline Zonisamide & 4138 & 4 & 7.1 & $(2.6-18.9)$ & 1916 & 2 & 1.3 & $(0.7-2.5)$ \\
\hline JADER & 430,587 & 80 & & & & & & \\
\hline \multicolumn{9}{|l|}{ Immunosuppressants } \\
\hline Cyclosporine & 8890 & 14 & 10.1 & $(5.7-17.9)$ & 5711 & 14 & 15.8 & $(8.9-28.2)$ \\
\hline Mycophenolate mofetil & 5320 & 3 & 3.1 & $(1.0-9.9)$ & 3060 & 1 & $-^{c}$ & \\
\hline \multicolumn{9}{|l|}{ Calcium Channel Blockers } \\
\hline Amlodipine & 30,451 & 18 & 3.8 & $(2.3-6.5)$ & 3025 & 18 & 41.3 & $(24.4-69.8)$ \\
\hline Benidipine & 3655 & 1 & $-^{c}$ & & 310 & 1 & $-^{c}$ & \\
\hline Nicardipine & 1755 & 5 & 16.3 & $(6.6-40.4)$ & 302 & 5 & 96.6 & $(38.8-240.5)$ \\
\hline Nifedipine & 10,350 & 8 & 4.5 & $(2.2-9.4)$ & 954 & 6 & 36.7 & $(15.9-84.6)$ \\
\hline \multicolumn{9}{|l|}{ Anticonvulsants } \\
\hline Carbamazepine & 7411 & 18 & 16.6 & $(9.8-28.1)$ & 5068 & 16 & 21.1 & $(12.2-36.4)$ \\
\hline Clobazam & 898 & 11 & 77.2 & $(40.7-146.4)$ & 239 & 10 & 268.4 & $(136.6-527.3)$ \\
\hline Diazepam & 3831 & 10 & 16.0 & $(8.2-31.0)$ & 733 & 6 & 47.9 & $(20.8-110.5)$ \\
\hline Gabapentin & 1222 & 2 & 9.0 & $(2.2-36.8)$ & 404 & 1 & $-^{c}$ & \\
\hline Levetiracetam & 1801 & 6 & 19.4 & $(8.4-44.6)$ & 1177 & 5 & 24.4 & $(9.9-60.5)$ \\
\hline Phenobarbital & 2622 & 12 & 28.9 & $(15.6-53.5)$ & 1027 & 9 & 53.5 & $(26.7-107.3)$ \\
\hline Phenytoin & 3712 & 27 & 59.0 & $(37.1-93.9)$ & 1667 & 22 & 98.9 & $(60.4-161.9)$ \\
\hline Primidone & 100 & 4 & 236.0 & $(84.7-657.8)$ & 25 & 4 & 1078.9 & $(361.8-3217.5)$ \\
\hline
\end{tabular}


Table 2 Number of reports and the reporting odds ratio for gingival hyperplasia by drugs (Continued)

\begin{tabular}{lllllllll}
\hline Topiramate & 536 & 1 & $-{ }^{c}$ & & 258 & 1 & $-^{c}$ & \\
Valproic acid & 8185 & 26 & 24.9 & $(15.6-39.8)$ & 2618 & 20 & 54.9 & $(33.0-91.2)$ \\
Zonisamide & 2492 & 12 & 30.5 & $(16.5-56.3)$ & 1073 & 8 & 44.8 & $(21.5-93.2)$ \\
\hline
\end{tabular}

a For FAERS, "Primary Suspect Drug" and "Secondary Suspect Drug" were analyzed

b For JADER, "Higiyaku" was analyzed

c Number of cases $<2$

involving CsA, everolimus, sirolimus, mycophenolate mofetil, amlodipine, nifedipine, carbamazepine, clobazam, levetiracetam, phenobarbital, phenytoin, primidone, topiramate, and valproic acid as PS or SS were 39.4 (95\% CI: 30.3-51.2), 4.2 (1.7-10.0), 6.6 (2.5-17.7), 13.1 (7.223.2), 94.8 (80.0-112.9), 57.9 (35.7-94.0), 15.1 (10.322.3), 65.4 (33.8-126.7), 6.5 (3.6-11.8), 19.7 (8.8-44.0), 65.4 (52.4-82.9), 56.5 (21.1-151.7), 2.9 (1.1-7.7), and 17.5 (12.6-24.4), respectively.

The JADER contained 430,587 reports submitted between April 2004 and November 2016. The lower limits of the ROR 95\% CI for CsA, amlodipine, nicardipine, nifedipine, carbamazepine, clobazam, diazepam, levetiracetam, phenobarbital, phenytoin, primidone, valproic acid, and zonisamide as suspected drug were all greater than one.

\section{Time-to-onset}

We evaluated data from the JADER database using timeto-onset analysis. The time-to-onset data and WSP are summarized in Fig. 1. The medians and quartile ranges for the onset day of DIGH after treatment with immunosuppressants, calcium channel blockers, and anticonvulsants were 71 (interquartile ranges: 22-120), 262 (76-442), and 37 (37-77) days, respectively. This time- to-onset profile shows that over $50 \%$ of DIGH were observed after $37-120$ days. The WSP $\beta$ and $95 \%$ CI of immunosuppressants, calcium channel blockers, and anticonvulsants were $1.41(0.31-3.82), 1.70(0.84-2.97)$, and 1.79 (1.23-2.44), respectively (Fig. 1 ). The WSP $\beta$ and $95 \%$ CI lower limit of anticonvulsants exceeded 1 , which describes a wear-out failure type, indicating a significant association between anticonvulsants and DIGH.

\section{Association rule mining}

We analyzed the JADER database using an association rule mining technique. Association rule mining was applied to the DIGH data using demographic data including age (22 items: < 10 y.o., child, adolescent, etc.), patient history in the all reported cases (8141 items: intellectual disability, cerebral palsy, epilepsy, etc.), administered drugs listed in the Table 1 (19 items: cyclosporine, everolimus, silorimus, etc.), and adverse event [1 item: gingival hypertrophy (PT code: 10018284)]. The apriori algorithm efficiently extracts sets of adverse events that occur more frequently than the minimum support threshold (defined as 0.00001 in this study), and generates sets of adverse events with the minimum confidence threshold (defined as 0.01 in this study). Furthermore, the maximum size of mined frequent itemsets (maxlen: a

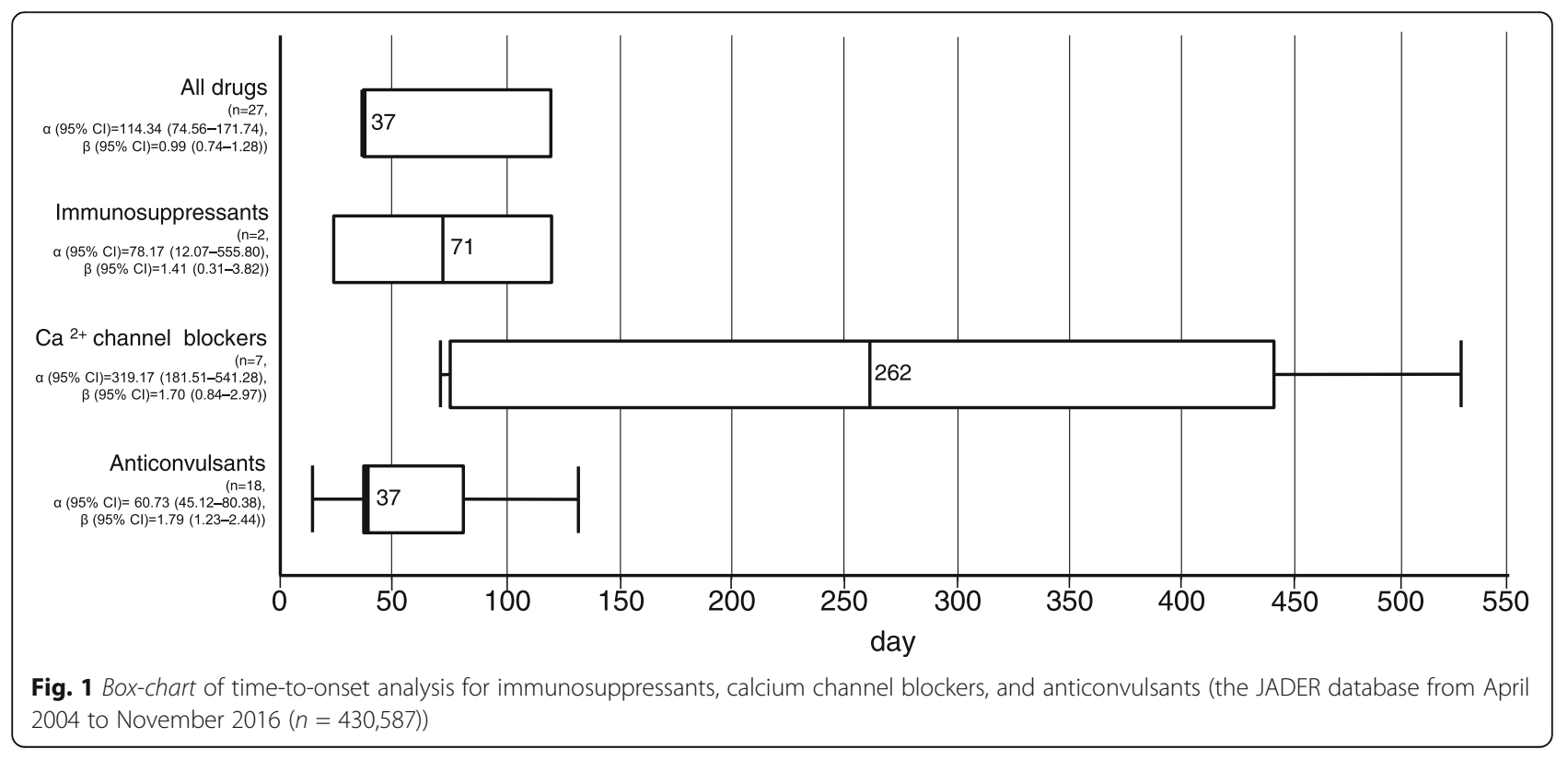


parameter in the arules package) was restricted to 3 . The result of the mining algorithm was a set of 44 rules (Tables 3 and 4). The support, confidence, and lift for each association rule are summarized in Tables 3 and 4; the association rules in descending order of the support are shown in Table 3, and in descending order of the lift are shown in Table 4. Anticonvulsants, especially phenytoin, demonstrated a high support value (Table 3, Fig. 2). The lift aspect of the association rules strength for anticonvulsants, especially phenytoin, carbamazepine, clobazam, and diazepam were high. The association rule of \{phenytoin, intellectual disability\} $\rightarrow$ \{gingival hypertrophy\} with high scores for lift and support were demonstrated (Table 3 (id [18]), Table 4 (id [4]), Fig. 2). For this rule, the values for support, confidence, and lift were 0.000021, 0.07, and 375.08 , respectively. The association rule of \{clobazam, diazepam\} $\rightarrow$ \{gingival hypertrophy\} demonstrated high scores for lift (Table 4 (id [3]), Fig. 2). The association rule of \{phenytoin, cerebral palsy\} $\rightarrow$ \{gingival hypertrophy\} and \{carbamazepine, cerebral palsy\} $\rightarrow$ \{gingival hypertrophy\} also demonstrated high scores for lift (Table 4 (id $[1,2])$, Fig. 2).

\section{Discussion}

Our results suggest that adverse-event signals of DIGH were detected for several drugs in the FAERS and JADER databases. The risk of DIGH is stated in the package inserts of amlodipine, phenytoin, and zonisamide in the US, and CsA, mycophenolate mofetil, amlodipine, benidipine, nifedipine, levetiracetam, phenytoin, topiramate, and valproic acid in Japan, which agrees with our results. Furthermore, we detected signals of DIGH for several drugs such as nicardipine, carbamazepine, clobazam, diazepam, phenobarbital, primidone, and zonisamide that have no adverse-event warning stated in their package inserts in Japan. A more detailed analysis focusing on these drugs should be the subject of future investigation.

Our study had some limitations that should be noted. SRSs are subject to numerous biases and confounders. Since the SRSs did not contain control populations, the ROR does not provide sufficient evidence on causality and should be considered exploratory in the context of signal detection $[5-7,16-21]$. The time-to-onset analysis using the WSP method allowed the detection of potential adverse events without requiring a control population [22, 23]. For this reason, we examined the time-toonset of DIGH using the WSP test.

To the best of our knowledge, no time-to-onset analyses of DIGH have been addressed using SRSs. The aim of the time-to-onset analysis was to obtain new information and compare the risks and onset profiles of DIGH for prescription drugs in the real world. The medians of the times-to-onset values for immunosuppressants and anticonvulsants were 71 and 37 days. The WSP $\beta$ of anticonvulsants was $1.79(1.23-2.44)$ and, so, the hazard was considered to increase over time (Fig. 1). DIGH induced by anticonvulsants was likely to be wear-out failure type. According to a report by Seymour et al. [24], phenytoin-induced gingival hyperplasia can occur within 3 months of drug use, which agrees with our results. These results also corresponded with those of previous reports. To alleviate DIGH, early countermeasures must be initiated. The effective treatments for DIGH are drug substitution or withdrawal, good oral hygiene practices such as plaque control [1], and surgical treatment. DIGH induced by anticonvulsants is clinically important because the number of therapies available for epilepsy has increased. Seizure control is the primary goal of epilepsy treatment [25] and therefore it is difficult to withdraw anticonvulsant drugs. Costa et al. [26] have reported that appropriate plaque control and early detection of periodontal disease is difficult, and that care and periodontal disease tend to worsen easily in patients with refractory epilepsy. The analysis results suggest that early monitoring of the gingival tissue following the observation of gingival hyperplasia in patients administered anticonvulsant agents is required to prevent aggravation of the condition.

The median onset of DIGH by calcium channel blockers was 262 days, which differed from those of anticonvulsants and immunosuppressants. Special attention should be paid to the possibility of DIGH occurrence with these drugs, and careful observation is recommended from 2 to 14 months.

The mechanism mediating the pathogenesis of medication-triggered connective tissue responses in the gingiva is still poorly understood. Some hypotheses have suggested the role of factors such as 1) fibroblasts [27-32], 2) inflammatory cytokines [30, 33-36], and 3) matrix metalloproteinase (MMP) synthesis [31]. CsA, nifedipine, and phenytoin promote the modeling of periodontal fibroblasts through the synthesis of gingival fibroblasts or inhibition of the decomposition of gingival fibroblasts [27-31]. Phenytoin may increase the level of translatable collagen mRNA in human gingival fibroblast [32], while CsA, nifedipine, and phenytoin enhance the synthesis of collagenous proteins in vitro [30, 33-36]. In the case of human gingival fibroblasts simultaneously exposed to nifedipine and interleukin-1 $\beta$ [33], an enhancement of collagenous protein synthesis was observed [33]. CsA may cause a decline in the secretion of MMP-1 and an accumulation of collagenous proteins [31]. The differences in these mechanisms may have affected the ROR value or time-to-onset profiles of each drug.

In the association rule mining approach, since the lift values of two combined items, \{phenytoin, intellectual disability\} were high, patients with intellectual disability 
Table 3 Association parameters of rules (sort by support)

\begin{tabular}{|c|c|c|c|c|c|c|}
\hline id & Ihs (left hand side, antecedent) & & rhs (right hand side, consequent) & support & confidence & lift \\
\hline [1] & \{epilepsy, phenytoin\} & $\rightarrow$ & \{gingival hypertrophy\} & 0.000044 & 0.02 & 85.21 \\
\hline [2] & $\{$ valproic acid, phenytoin\} & $\rightarrow$ & \{gingival hypertrophy\} & 0.000044 & 0.02 & 125.83 \\
\hline [3] & \{carbamazepine, phenytoin\} & $\rightarrow$ & \{gingival hypertrophy\} & 0.000030 & 0.02 & 119.21 \\
\hline [4] & $\{c e r e b r a l$ palsy\} & $\rightarrow$ & \{gingival hypertrophy\} & 0.000028 & 0.02 & 82.79 \\
\hline [5] & \{epilepsy, cerebral palsy\} & $\rightarrow$ & \{gingival hypertrophy\} & 0.000028 & 0.05 & 233.00 \\
\hline [6] & \{carbamazepine, valproic acid\} & $\rightarrow$ & \{gingival hypertrophy\} & 0.000028 & 0.01 & 55.27 \\
\hline [7] & $\{c l o b a z a m\}$ & $\rightarrow$ & \{gingival hypertrophy\} & 0.000026 & 0.01 & 62.79 \\
\hline [8] & $\{$ intellectual disability\} & $\rightarrow$ & \{gingival hypertrophy\} & 0.000023 & 0.01 & 60.38 \\
\hline [9] & $\{$ valproic acid, cerebral palsy\} & $\rightarrow$ & \{gingival hypertrophy\} & 0.000023 & 0.05 & 244.10 \\
\hline [10] & \{epilepsy, intellectual disability\} & $\rightarrow$ & \{gingival hypertrophy\} & 0.000023 & 0.03 & 130.43 \\
\hline [11] & \{valproic acid, intellectual disability\} & $\rightarrow$ & \{gingival hypertrophy\} & 0.000023 & 0.03 & 152.11 \\
\hline$[12]$ & \{zonisamide, phenobarbital\} & $\rightarrow$ & \{gingival hypertrophy\} & 0.000023 & 0.04 & 183.73 \\
\hline [13] & $\{$ epilepsy, phenobarbital\} & $\rightarrow$ & \{gingival hypertrophy\} & 0.000023 & 0.01 & 73.54 \\
\hline [14] & $\{$ valproic acid, phenobarbital\} & $\rightarrow$ & \{gingival hypertrophy\} & 0.000023 & 0.02 & 78.38 \\
\hline$[15]$ & $\{<10$ y.o., phenobarbital $\}$ & $\rightarrow$ & \{gingival hypertrophy\} & 0.000023 & 0.02 & 115.45 \\
\hline$[16]$ & $\{$ zonisamide, valproic acid\} & $\rightarrow$ & \{gingival hypertrophy\} & 0.000023 & 0.01 & 70.41 \\
\hline$[17]$ & $\{$ diazepam, valproic acid\} & $\rightarrow$ & \{gingival hypertrophy\} & 0.000023 & 0.02 & 123.52 \\
\hline [18] & \{phenytoin, intellectual disability\} & $\rightarrow$ & \{gingival hypertrophy\} & 0.000021 & 0.07 & 375.08 \\
\hline [19] & $\{<10$ y.o., zonisamide $\}$ & $\rightarrow$ & \{gingival hypertrophy\} & 0.000021 & 0.03 & 172.14 \\
\hline [20] & $\{$ diazepam, epilepsy\} & $\rightarrow$ & \{gingival hypertrophy\} & 0.000021 & 0.03 & 137.71 \\
\hline$[21]$ & \{carbamazepine, diazepam\} & $\rightarrow$ & \{gingival hypertrophy\} & 0.000021 & 0.03 & 142.39 \\
\hline$[22]$ & \{carbamazepine, cerebral palsy\} & $\rightarrow$ & \{gingival hypertrophy\} & 0.000019 & 0.08 & 394.31 \\
\hline$[23]$ & $\{c l o b a z a m$, valproic acid\} & $\rightarrow$ & \{gingival hypertrophy\} & 0.000019 & 0.01 & 76.22 \\
\hline$[24]$ & \{diazepam, phenytoin\} & $\rightarrow$ & \{gingival hypertrophy\} & 0.000019 & 0.04 & 206.07 \\
\hline$[25]$ & $\{10-19$ years of age, epilepsy\} & $\rightarrow$ & \{gingival hypertrophy\} & 0.000019 & 0.01 & 52.51 \\
\hline$[26]$ & $\{<10$ y.o., epilepsy $\}$ & $\rightarrow$ & \{gingival hypertrophy\} & 0.000019 & 0.01 & 59.78 \\
\hline$[27]$ & $\{<10$ y.o., valproic acid $\}$ & $\rightarrow$ & \{gingival hypertrophy\} & 0.000019 & 0.01 & 58.17 \\
\hline$[28]$ & \{phenytoin, cerebral palsy\} & $\rightarrow$ & \{gingival hypertrophy\} & 0.000016 & 0.12 & 618.66 \\
\hline [29] & $\{c l o b a z a m$, phenytoin\} & $\rightarrow$ & \{gingival hypertrophy\} & 0.000016 & 0.03 & 153.34 \\
\hline$[30]$ & $\{c l o b a z a m$, diazepam\} & $\rightarrow$ & \{gingival hypertrophy\} & 0.000016 & 0.07 & 377.71 \\
\hline$[31]$ & \{phenytoin, phenobarbital\} & $\rightarrow$ & \{gingival hypertrophy\} & 0.000016 & 0.01 & 75.70 \\
\hline$[32]$ & $\{10-19$ years of age, phenytoin $\}$ & $\rightarrow$ & \{gingival hypertrophy\} & 0.000016 & 0.04 & 199.35 \\
\hline [33] & \{periodontitis\} & $\rightarrow$ & \{gingival hypertrophy\} & 0.000014 & 0.01 & 71.69 \\
\hline [34] & \{zonisamide, intellectual disability\} & $\rightarrow$ & \{gingival hypertrophy\} & 0.000014 & 0.05 & 256.30 \\
\hline [35] & \{clobazam, zonisamide\} & $\rightarrow$ & \{gingival hypertrophy\} & 0.000014 & 0.02 & 106.42 \\
\hline$[36]$ & $\{c l o b a z a m, ~ e p i l e p s y\}$ & $\rightarrow$ & \{gingival hypertrophy\} & 0.000014 & 0.01 & 61.88 \\
\hline$[37]$ & \{carbamazepine, clobazam\} & $\rightarrow$ & \{gingival hypertrophy\} & 0.000014 & 0.02 & 97.02 \\
\hline [38] & $\{$ carbamazepine, phenobarbital\} & $\rightarrow$ & \{gingival hypertrophy\} & 0.000014 & 0.02 & 79.89 \\
\hline [39] & \{zonisamide, phenytoin\} & $\rightarrow$ & \{gingival hypertrophy\} & 0.000014 & 0.02 & 89.67 \\
\hline$[40]$ & \{phenobarbital, cerebral palsy\} & $\rightarrow$ & \{gingival hypertrophy\} & 0.000012 & 0.04 & 194.17 \\
\hline [41] & $\{<10$ y.o., cerebral palsy $\}$ & $\rightarrow$ & \{gingival hypertrophy\} & 0.000012 & 0.02 & 107.69 \\
\hline [42] & $\{c l o b a z a m$, phenobarbital\} & $\rightarrow$ & \{gingival hypertrophy\} & 0.000012 & 0.03 & 141.60 \\
\hline$[43]$ & $\{<10$ y.o., clobazam $\}$ & $\rightarrow$ & \{gingival hypertrophy\} & 0.000012 & 0.02 & 111.44 \\
\hline$[44]$ & \{clonazepam, phenytoin\} & $\rightarrow$ & \{gingival hypertrophy\} & 0.000012 & 0.02 & 114.93 \\
\hline
\end{tabular}


Table 4 Association parameters of rules (sort by lift)

\begin{tabular}{|c|c|c|c|c|c|c|}
\hline id & Ihs (left hand side, antecedent) & & rhs (right hand side, consequent) & support & confidence & lift \\
\hline [1] & \{phenytoin, cerebral palsy\} & $\rightarrow$ & \{gingival hypertrophy\} & 0.000016 & 0.12 & 618.66 \\
\hline [2] & \{carbamazepine, cerebral palsy\} & $\rightarrow$ & \{gingival hypertrophy\} & 0.000019 & 0.08 & 394.31 \\
\hline [3] & $\{c l o b a z a m$, diazepam\} & $\rightarrow$ & \{gingival hypertrophy\} & 0.000016 & 0.07 & 377.71 \\
\hline [4] & \{phenytoin, intellectual disability\} & $\rightarrow$ & \{gingival hypertrophy\} & 0.000021 & 0.07 & 375.08 \\
\hline [5] & \{zonisamide, intellectual disability\} & $\rightarrow$ & \{gingival hypertrophy\} & 0.000014 & 0.05 & 256.30 \\
\hline [6] & $\{$ valproic acid, cerebral palsy\} & $\rightarrow$ & \{gingival hypertrophy\} & 0.000023 & 0.05 & 244.10 \\
\hline [7] & \{epilepsy, cerebral palsy\} & $\rightarrow$ & \{gingival hypertrophy\} & 0.000028 & 0.05 & 233.00 \\
\hline [8] & \{diazepam, phenytoin\} & $\rightarrow$ & \{gingival hypertrophy\} & 0.000019 & 0.04 & 206.07 \\
\hline [9] & $\{10-19$ years of age, phenytoin $\}$ & $\rightarrow$ & \{gingival hypertrophy\} & 0.000016 & 0.04 & 199.35 \\
\hline [10] & \{phenobarbital, cerebral palsy\} & $\rightarrow$ & \{gingival hypertrophy\} & 0.000012 & 0.04 & 194.17 \\
\hline [11] & \{zonisamide, phenobarbital\} & $\rightarrow$ & \{gingival hypertrophy\} & 0.000023 & 0.04 & 183.73 \\
\hline$[12]$ & $\{<10$ y.o., zonisamide $\}$ & $\rightarrow$ & \{gingival hypertrophy\} & 0.000021 & 0.03 & 172.14 \\
\hline [13] & \{clobazam, phenytoin\} & $\rightarrow$ & \{gingival hypertrophy\} & 0.000016 & 0.03 & 153.34 \\
\hline [14] & \{valproic acid, intellectual disability\} & $\rightarrow$ & \{gingival hypertrophy\} & 0.000023 & 0.03 & 152.11 \\
\hline$[15]$ & \{carbamazepine, diazepam\} & $\rightarrow$ & \{gingival hypertrophy\} & 0.000021 & 0.03 & 142.39 \\
\hline$[16]$ & $\{c l o b a z a m$, phenobarbital\} & $\rightarrow$ & \{gingival hypertrophy\} & 0.000012 & 0.03 & 141.60 \\
\hline$[17]$ & $\{$ diazepam, epilepsy\} & $\rightarrow$ & \{gingival hypertrophy\} & 0.000021 & 0.03 & 137.71 \\
\hline [18] & \{epilepsy, intellectual disability\} & $\rightarrow$ & \{gingival hypertrophy\} & 0.000023 & 0.03 & 130.43 \\
\hline [19] & \{valproic acid, phenytoin\} & $\rightarrow$ & \{gingival hypertrophy\} & 0.000044 & 0.02 & 125.83 \\
\hline [20] & $\{$ diazepam, valproic acid\} & $\rightarrow$ & \{gingival hypertrophy\} & 0.000023 & 0.02 & 123.52 \\
\hline$[21]$ & \{carbamazepine, phenytoin\} & $\rightarrow$ & \{gingival hypertrophy\} & 0.000030 & 0.02 & 119.21 \\
\hline$[22]$ & $\{<10$ y.o., phenobarbital $\}$ & $\rightarrow$ & \{gingival hypertrophy\} & 0.000023 & 0.02 & 115.45 \\
\hline$[23]$ & \{clonazepam, phenytoin\} & $\rightarrow$ & \{gingival hypertrophy\} & 0.000012 & 0.02 & 114.93 \\
\hline$[24]$ & $\{<10$ y.o., clobazam $\}$ & $\rightarrow$ & \{gingival hypertrophy\} & 0.000012 & 0.02 & 111.44 \\
\hline$[25]$ & $\{<10$ y.o., cerebral palsy $\}$ & $\rightarrow$ & \{gingival hypertrophy\} & 0.000012 & 0.02 & 107.69 \\
\hline [26] & $\{c l o b a z a m$, zonisamide\} & $\rightarrow$ & \{gingival hypertrophy\} & 0.000014 & 0.02 & 106.42 \\
\hline$[27]$ & \{carbamazepine, clobazam\} & $\rightarrow$ & \{gingival hypertrophy\} & 0.000014 & 0.02 & 97.02 \\
\hline$[28]$ & \{zonisamide, phenytoin\} & $\rightarrow$ & \{gingival hypertrophy\} & 0.000014 & 0.02 & 89.67 \\
\hline [29] & \{epilepsy, phenytoin\} & $\rightarrow$ & \{gingival hypertrophy\} & 0.000044 & 0.02 & 85.21 \\
\hline$[30]$ & $\{c e r e b r a l$ palsy\} & $\rightarrow$ & \{gingival hypertrophy\} & 0.000028 & 0.02 & 82.79 \\
\hline$[31]$ & \{carbamazepine, phenobarbital\} & $\rightarrow$ & \{gingival hypertrophy\} & 0.000014 & 0.02 & 79.89 \\
\hline$[32]$ & $\{$ valproic acid, phenobarbital\} & $\rightarrow$ & \{gingival hypertrophy\} & 0.000023 & 0.02 & 78.38 \\
\hline [33] & $\{c l o b a z a m$, valproic acid $\}$ & $\rightarrow$ & \{gingival hypertrophy\} & 0.000019 & 0.01 & 76.22 \\
\hline$[34]$ & \{phenytoin, phenobarbital\} & $\rightarrow$ & \{gingival hypertrophy\} & 0.000016 & 0.01 & 75.70 \\
\hline [35] & $\{$ epilepsy, phenobarbital\} & $\rightarrow$ & \{gingival hypertrophy\} & 0.000023 & 0.01 & 73.54 \\
\hline$[36]$ & \{periodontitis\} & $\rightarrow$ & \{gingival hypertrophy\} & 0.000014 & 0.01 & 71.69 \\
\hline$[37]$ & $\{z o n i s a m i d e$, valproic acid\} & $\rightarrow$ & \{gingival hypertrophy\} & 0.000023 & 0.01 & 70.41 \\
\hline [38] & $\{c l o b a z a m\}$ & $\rightarrow$ & \{gingival hypertrophy\} & 0.000026 & 0.01 & 62.79 \\
\hline [39] & $\{c l o b a z a m$, epilepsy\} & $\rightarrow$ & \{gingival hypertrophy\} & 0.000014 & 0.01 & 61.88 \\
\hline$[40]$ & \{intellectual disability\} & $\rightarrow$ & \{gingival hypertrophy\} & 0.000023 & 0.01 & 60.38 \\
\hline [41] & $\{<10$ y.o., epilepsy $\}$ & $\rightarrow$ & \{gingival hypertrophy\} & 0.000019 & 0.01 & 59.78 \\
\hline [42] & $\{<10$ y.o., valproic acid $\}$ & $\rightarrow$ & \{gingival hypertrophy\} & 0.000019 & 0.01 & 58.17 \\
\hline$[43]$ & \{carbamazepine, valproic acid\} & $\rightarrow$ & \{gingival hypertrophy\} & 0.000028 & 0.01 & 55.27 \\
\hline$[44]$ & $\{10-19$ years of age, epilepsy\} & $\rightarrow$ & \{gingival hypertrophy\} & 0.000019 & 0.01 & 52.51 \\
\hline
\end{tabular}




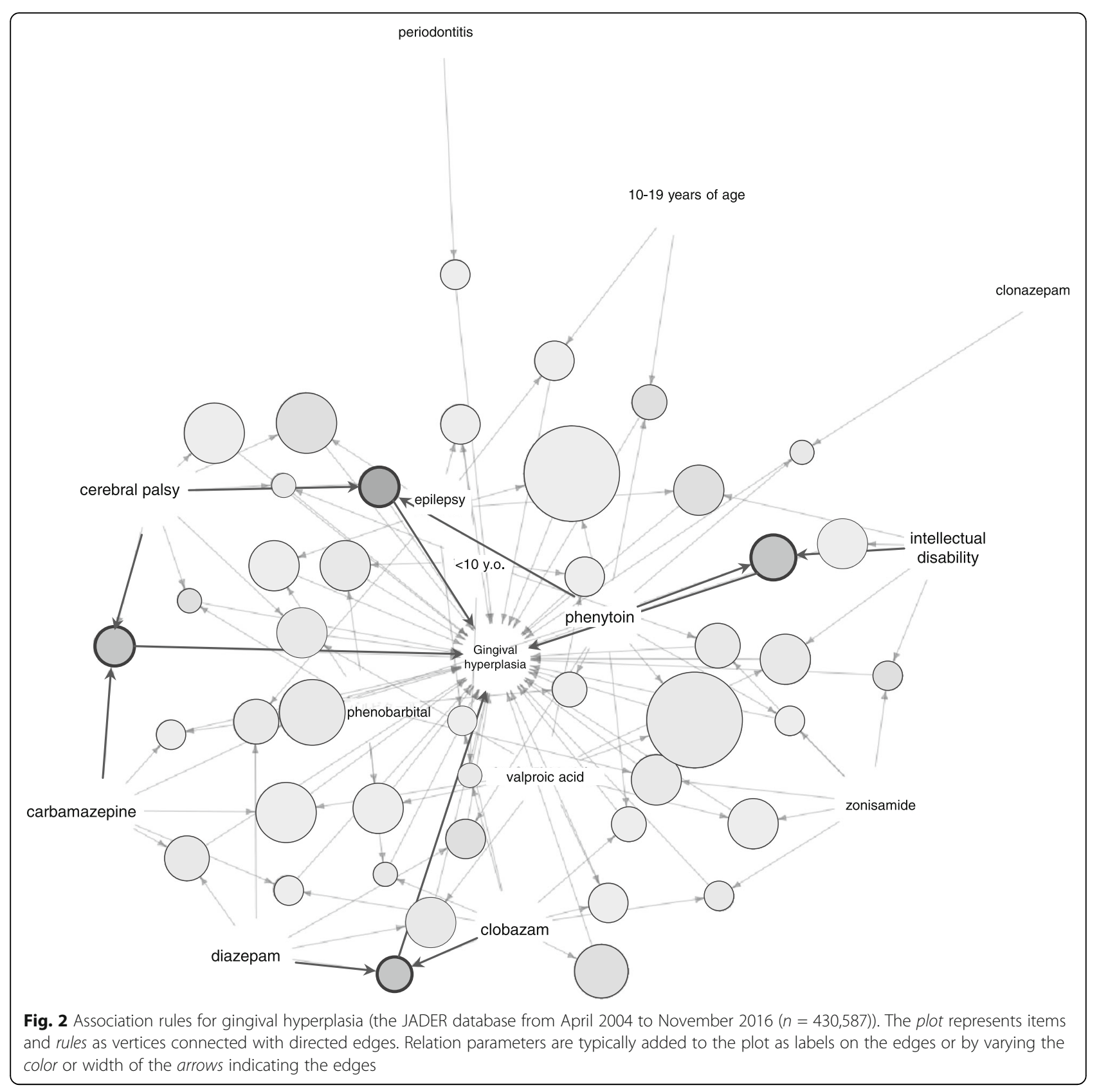

have a potential risk of DIGH following treatment with anticonvulsants. Therefore, patients with intellectual disabilities should be monitored carefully. The lift values of two combined items \{diazepam, clobazam\}, which were greater than that of one value were also high enough to suggests a strong association. The support value was low, and these data suggest that each association was strong, although the expression rates were low. This information suggests that polypharmacy with anticonvulsants may increase the risk of DIGH. Since Harpaz et al. addressed the issue of confounding factors when applying the association rule mining approach, our obtained association rules might be tabulated independently in the future to evaluate the confounding factors related to DIGH [3].

Patients who were administered calcium channel blockers such as nifedipine or amlodipine demonstrated high drug levels in their gingival crevicular fluid and were likely to be exposed to high levels of these drugs $[37,38]$. DIGH was observed with CsA treatment in $25-30 \%$ and $\geq 70 \%$ of adults and pediatric patients, respectively [1]. Coadministration of medications with CsA increased the risk of CsA-induced gingival hyperplasia [39], although, the relationship between the dosage, duration of therapy, age, and sex is still not clear. Unfortunately, cases reported in the SRS database do not always contain sufficient 
information on patient background, drug dosage, drugdrug interactions and mode of administration to allow proper evaluation. Considering the causality constraints of the current analysis, further epidemiological studies are recommended.

\section{Conclusions}

This study was the first to evaluate the incidences of DIGH using SRSs. Despite the limitations inherent to SRS, we identified the risk of DIGH induced by anticonvulsants, immunosuppressants, and calcium channel blockers. We demonstrated the potential risk of DIGH following the long-term use of calcium channel blocker for approximately 260 days. The association rule mining results suggest that patients with intellectual disabilities administered phenytoin, should be monitored carefully. We recommend that patients who experience symptoms related to gingival hyperplasia should be closely monitored and advised to adhere to an appropriate care plan for oral hygiene. Finally, it is our hope that these data will update the information available to clinicians and be potentially useful for improving the management of DIGH.

\section{Abbreviations \\ C: Concomitant; Cl: Confidence Interval; CsA: Cyclosporine; DIGH: Drug- induced gingival hyperplasia; FAERS: US Food and Drug Administration Adverse Event Reporting System; FDA: Food and Drug Administration; I: Interacting; JADER: Japanese Adverse Drug Event Report; MedDRA: Medical Dictionary for Regulatory Activities; MMP: Matrix metalloproteinase; PMDA: The Pharmaceuticals and Medical Devices Agency; PS: Primary Suspect; PT: Preferred Term; QOL: Quality of life; ROR: Reporting Odds Ratio; SRS: Spontaneous Reporting System; SS: Secondary Suspect; WSP: Weibull Shape Parameter}

\section{Acknowledgements}

Not applicable.

\section{Funding}

This research was partially supported by JSPS KAKENHI Grant number, 24360126 and $17 K 08452$.

\section{Availability of data and materials}

The dataset supporting the conclusions of this article is included within the article.

\begin{abstract}
Authors' contributions
$\mathrm{HH}$ and $\mathrm{MN}$ conceived of the study, participated in its design and coordination, and drafted the manuscript. JA conceived of the study, participated in its design, helped the statistical analysis, and drafted the manuscript. YH, TM, SS, YM performed the statistical analysis. SH, AF, and MN helped to interpret of data. YK and TO helped to interpretation of data, and participated in its design of the study. All authors read and approved the final manuscript.
\end{abstract}

\section{Ethics approval and consent to participate} Not applicable.

\section{Consent for publication}

Not applicable.

\section{Competing interests}

JA is an employee of Medical Database Co., Ltd. TO is an employee of Ace Pharmacy, Seiyou Trading Co., Ltd. The other authors have no conflict of interest.

\section{Publisher's Note}

Springer Nature remains neutral with regard to jurisdictional claims in published maps and institutional affiliations.

\section{Author details}

${ }^{1}$ Laboratory of Drug Informatics, Gifu Pharmaceutical University, 1-25-4 Daigaku-nishi, Gifu 501-1196, Japan. ${ }^{2}$ Medical Database Co., Ltd, 3-11-10 Higashi, Shibuya-ku, Tokyo 150-0011, Japan. ${ }^{3}$ Ace Pharmacy, Seiyou Trading Co., Ltd, 1343 Funatsuke Nakashiro, Yoro-cho, Gifu 503-1382, Japan. ${ }^{4}$ United Graduate School of Drug Discovery and Medical Information Sciences, Gifu University, 1-1 Yanagido, Gifu 501-1194, Japan.

Received: 27 March 2017 Accepted: 9 July 2017

Published online: 19 July 2017

\section{References}

1. Dongari-Bagtzoglou A. Informational paper: drug-associated gingival enlargement. J Periodontol. 2004;75:1424-31.

2. Fujiwara M, Kawasaki Y, Yamada H. A Pharmacovigilance approach for postMarketing in Japan Using the Japanese adverse drug event report (JADER) database and association analysis. Plos One. 2016;11:e0154425.

3. Harpaz R, Chase HS, Friedman C. Mining multi-item drug adverse effect associations in spontaneous reporting systems. BMC Bioinformatics. 2010;11:S7.

4. Yildirim P. Association patterns in open data to explore ciprofloxacin adverse events. Appl Clin Inform. 2015;6:728-47.

5. Hashiguchi M, Imai S, Uehara K, Maruyama J, Shimizu M, Mochizuki M. Factors affecting the timing of signal detection of adverse drug reactions. Plos One. 2015;10:e0144263.

6. Poluzzi E, Raschi E, Piccinni C, De Ponti F. Data mining techniques in Pharmacovigilance: analysis of the publicly accessible FDA adverse event reporting system (AERS). INTECH. 2012:265-302. doi:10.5772/50095.

7. Nakamura M, Umetsu R, Abe J, Matsui T, Ueda N, Kato Y, et al. Analysis of the time-to-onset of osteonecrosis of jaw with bisphosphonate treatment using the data from a spontaneous reporting system of adverse drug events. J Pharm Health Care Sci. 2015;1:34.

8. Abe J, Umetsu R, Mataki K, Kato Y, Ueda N, Nakayama Y, et al. Analysis of Stevens-Johnson syndrome and toxic epidermal necrolysis using the Japanese adverse drug event report database. J Pharm Health Care Sci. 2016;2:14.

9. Sasaoka S, Matsui T, Hane Y, Abe J, Ueda N, Motooka Y, et al. Time-to-onset analysis of drug-induced long QT syndrome based on a spontaneous reporting system for adverse drug events. Plos One. 2016:11:e0164309.

10. Sasaoka S, Matsui T, Hane Y, Abe J, Ueda N, Motooka Y, et al. Evaluation of the association of hand-foot syndrome with anticancer drugs using the US Food and Drug Administration adverse event reporting system (FAERS) and Japanese adverse drug event report (JADER) databases. Yakugaku Zasshi. 2016;136:507-15.

11. Leroy F, Dauxois JY, Théophile H, Haramburu F, Tubert-Bitter P. Estimating time-to-onset of adverse drug reactions from spontaneous reporting databases. BMC Med Res Methodol. 2014;14:17.

12. Agrawal R, Srikant R. Fast Algorithms for Mining Association Rules in Large Databases. Proceedings of the 20th International Conference on Very Large Data Bases. 1994:7:478-99.

13. Agrawal R, Imielinski T, Swami A. Mining association rules between sets of items in large databases. Special Interenst Group on Management of Data. 1993;22:207-16

14. Zhu AL, Li J, Leong TY. Automated knowledge extraction for decision model construction: a data mining approach. AMIA Ann Symp Proc. 2003; 2003:758-76.

15. Hahsler M, Grün B, Hornik K. A computational environment for mining association rules and frequent item sets. J Stat Soft. 2005;14:15.

16. Suzuki Y, Suzuki H, Umetsu R, Uranishi H, Abe J, Nishibata Y, et al. Analysis of the interaction between clopidogrel, aspirin, and proton pump inhibitors using the FDA adverse event reporting system database. Biol Pharm Bull. 2015;38:680-6. 
17. Abe J, Umetsu R, Kato Y, Ueda N, Nakayama Y, Suzuki Y, et al. Evaluation of Dabigatran- and Warfarin-associated hemorrhagic events using the FDAadverse event reporting system database stratified by age. Int J Med Sci. 2015:12:312-21.

18. Ueda N, Umetsu R, Abe J, Kato Y, Nakayama Y, Kato Z, et al. Analysis of neuropsychiatric adverse events in patients treated with Oseltamivir in spontaneous adverse event reports. Biol Pharm Bull. 2015;38:1638-44.

19. Umetsu R, Nishibata Y, Abe J, Suzuki Y, Hara H, Nagasawa $H$, et al. Evaluation of the association between the use of oral anti-hyperglycemic agents and hypoglycemia in Japan by data mining of the Japanese adverse drug event report (JADER) database. Yakugaku Zasshi. 2014;134:299-304.

20. Kato Y, Umetsu R, Hosoya N, Ueda N, Abe J, Nakayama Y, et al. Analysis of licorice-induced pseudoaldosteronism in the Japanese adverse drug event report database. Tradit Kampo Med. 2016;3:63-70.

21. Kato Y, Umetsu R, Abe J, Ueda N, Nakayama Y, Kinosada Y, et al. Hyperglycemic adverse events following antipsychotic drug administration in spontaneous adverse event reports. J Pharm Health Care Sci. 2015;1:15.

22. Yamada M, Handa J. Comparison of the onset time profile among the interferon formulations in adverse drug reaction of suicide- or diabetes related. Jpn J Pharmacoepidemiol. 2014;19:23-30.

23. Sauzet O, Carvajal A, Escudero A, Molokhia M, Cornelius VR. Illustration of the weibull shape parameter signal detection tool using electronic healthcare record data. Drug Saf. 2013;36:995-1006.

24. Seymour RA, Ellis JS, Thomason JM. Risk factors for drug-induced gingival overgrowth. J Clin Periodontol. 2000;27:217-23.

25. Johnson EK, Jones JE, Seidenberg M, Hermann BP. The relative impact of anxiety, depression, and clinical seizure features on health-related quality of life in epilepsy. Epilepsia. 2004;45:544-50.

26. Costa AL, Yasuda CL, Shibasaki W, Nahás-Scocate AC, de Freitas CF, Carvalho $\mathrm{PE}$, et al. The association between periodontal disease and seizure severity in refractory epilepsy patients. Seizure. 2014;23:227-30.

27. Hassell TM, Page RC, Narayanan AS, Cooper CG. Diphenylhydantoin (Dilantin) gingival hyperplasia: drug-induced abnormality of connective tissue. Proc Natl Acad Sci. 1976;73:2909-12.

28. Chung Y, Fu E. Crosstalk between Shh and TGF- $\beta$ signaling in cyclosporineenhanced cell proliferation in human gingival fibroblasts. Plos One. 2013;8: e70128.

29. Montebugnoli L, Bernardi F. Cyclosporin a effects on extra oral connective tissue in heart transplanted patients. J Int Acad Periodontol. 1999;1:91-4.

30. Morton RS, Dongari-Bagtzoglou Al. Regulation of gingival fibroblast interleukin-6 secretion by cyclosporine a. J Periodontol. 1999;70:1464-71.

31. Bolzani G, Coletta RD, Junior HM, Almeida OP, Graner E. Cyclosporin a inhibits production and activity of matrix metalloproteinases by gingival fibroblasts. J Periodontal Res. 2000;35:51-8.

32. Deshmukh SN, Dive AM, Moharil R, Munde P. Enigmatic insight into collagen. J Oral Maxillofac Pathol. 2016;20:276-83.

33. Johnson RB, Zebrowski EJ, Dai X. Synergistic enhancement of collagenous protein synthesis by human gingival fibroblasts exposed to nifedipine and interleukin-1-beta in vitro. J Oral Pathol Med. 2000;29:8-12.

34. Williamson MS, Miller EK, Plemons J, Rees T, lacopino AM. Cyclosporine a upregulates interleukin-6 gene expression in human gingiva: possible mechanism for gingival overgrowth. J Periodontol. 1994;65:895-903.

35. Duncan MR, Berman B. Stimulation of collagen and Glycosaminoglycan production in cultured human adult dermal fibroblasts by recombinant human interleukin 6. J Invest Dermatol. 1991;97:686-92.

36. Myrillas TT, Linden GJ, Marley JJ, Irwin CR. Cyclosporin a regulates interleukin-1 beta and interleukin-6 expression in gingiva: implications for gingival overgrowth. J Periodontol. 1999;70:294-300.

37. Seymour RA, Preshaw PM, Thomason JM, Ellis JS, Steele JG. Cardiovascular diseases and periodontology. J Clin Periodontol. 2003;30:279-92.

38. Ellis JS, Seymour RA, Monkman SC, Idle JR. Gingival sequestration of nifedipine in nifedipine-induced gingival overgrowth. Lancet. 1992;339: 1382-3.

39. Bartoli F, Castronovo G, Stabile A. Risk factors conditioning the incidence and severity of cyclosporine A-induced gingival overgrowth and methods of prevention. Minerva Stomatol. 2004;53:165-70.

\section{Submit your next manuscript to BioMed Central and we will help you at every step:}

- We accept pre-submission inquiries

- Our selector tool helps you to find the most relevant journal

- We provide round the clock customer support

- Convenient online submission

- Thorough peer review

- Inclusion in PubMed and all major indexing services

- Maximum visibility for your research

Submit your manuscript at www.biomedcentral.com/submit 